\title{
Undergraduate experiments in optics employing a fiber optic version of the Mach-Zehnder interferometer
}

\section{Adonis Flores, Marlo Flores, Kees Karremans, Ben Zuidberg}

Adonis Flores, Marlo Flores, Kees Karremans, Ben Zuidberg, "Undergraduate experiments in optics employing a fiber optic version of the Mach-Zehnder interferometer," Proc. SPIE 4588, Seventh International Conference on Education and Training in Optics and Photonics, (28 May 2002); doi: $10.1117 / 12.468678$

SPIE Event: Education and Training in Optics and Photonics 2001, 2001, Singapore, Singapore 


\title{
Undergraduate Experiments in Optics Employing a Fiber Optic Version of the Mach-Zehnder Interferometer
}

\author{
Adonis Flores, Marlo Flores, Kees Karremans and Ben Zuidberg \\ Optoelectronics Laboratory, Department of Physics, University of San Carlos, \\ Cebu City 6000, Philippines
}

\begin{abstract}
Interferometry (the use of interference phenomena) provides ample opportunities for measurements in various areas of physics, particularly in optics. In an interferometer, light from a single source is split into two beams that travel along different paths. The beams are recombined to produce an interference pattern that can be used to detect changes in the optical path length in one of the two arms. Here we report about the use of a fiber optic version of the Mach-Zehnder interferometer in measurements of the index of refraction of water and air.

The open air version of the Mach-Zehnder interferometer employs two beam splitters and two highly reflective mirrors. This open air version is difficult to align and sensitive to environmental disturbance. In our fiber optic version we have replaced one beamsplitter and two mirrors by a bidirectional coupler supplied with single mode fibers. This replacement greatly simplified the operation of the interferometer. A stable interference pattern could quite easily be obtained. The simplified operation allowed the introduction of the instrument in our BS program. This year two students performed highly accurate measurements on the index of refraction of various fluids (water, air) for their graduate project. Recently the instrument has been introduced in the regular laboratory classes.
\end{abstract}

\section{INTRODUCTION}

In 1996 the University of San Carlos (USC), Cebu City, Philippines embarked on a new BS Applied Physics program. The program should serve the needs of local and regional industries. Links with industries and government agencies were established in order to ensure that the program was brought in line with the local and regional needs for physics graduates. The Mactan Free Economic Zone (located close to Cebu) accommodates a number of companies that are manufacturing optical and electronic products. Hence optoelectronics was chosen as one of the focal points for research under the new program.

The final course in the BS Applied Physics program is Physics Research. The students work for about one month fulltime on a particular research topic. Working at BS level imposes some restrictions on the choice of the topics for research, apart from the financial constraints that may be there. Below we report about a successful introduction of a fiber optic version of the Mach-Zehnder interferometer in our BS program.

In an interferometer light from a single source is split into two beams that travel along different paths. The beams are then recombined to form an interference pattern. Changes in the optical path length in one of the two arms will produce a shift of the interference pattern. This enables the use of the interferometer in measurements on the index of refraction.

The open air version of the Mach-Zehnder interferometer employs two beamsplitters and two highly reflective mirrors.. The operation of the open air version of the interferometer is a tedious and painstaking job and requires very good skills in optics. The replacement of one beamsplitter and two mirrors by a bidirectional coupler supplied with single mode fibers greatly simplifies the operation of the interferometer and allows the introduction in experiments at BS level. Over the past two years two BS graduate projects have been done using the fiber optic version of the Mach Zehnder interferometer. In 2000 two students constructed the fiber optic version and tested its potential in various applications (temperature and pressure sensor). In 2001 two students used the interferometer for measurements on the index of refraction of various fluids (water, air). The highly accurate results gave clear evidence for the high potential of the instrument when used as a refractometer. The relatively simple operation and the limited cost allow introduction in the BS laboratory classes. At USC we have recently introduced the fiber optic version of the $\mathrm{MZ}$ interferometer in the BS laboratory class. The various factors (polarization, intensity distribution,), that influence its operation, enable the use of the instrument in different experiments in optics. 


\section{THEORY}

In the measurement of the index of refraction using the Mach-Zehnder interferometer, a sample of thickness $d$ with index of refraction $n_{\mathrm{o}}$ is inserted in one of the arms of the interferometer (Figure 1). The insertion of this sample increases the optical path length in this arm due to the fact that light travels more slowly in a medium ${ }^{1}$ as compared to air. The optical path length in the sample is equal to $n_{\mathrm{o}} d$. When the temperature and/or he density of the sample changes, the index of refraction will change to $n$. This corresponds to a change in the optical path length of $\left(n-n_{0}\right) d$. This will result in a shift of the fringe pattern by $\Delta m$ fringes where

$$
\Delta m=\left(n-n_{\mathrm{o}}\right) d / \lambda
$$

where $\lambda$ is the wavelength of the light ${ }^{1}$.

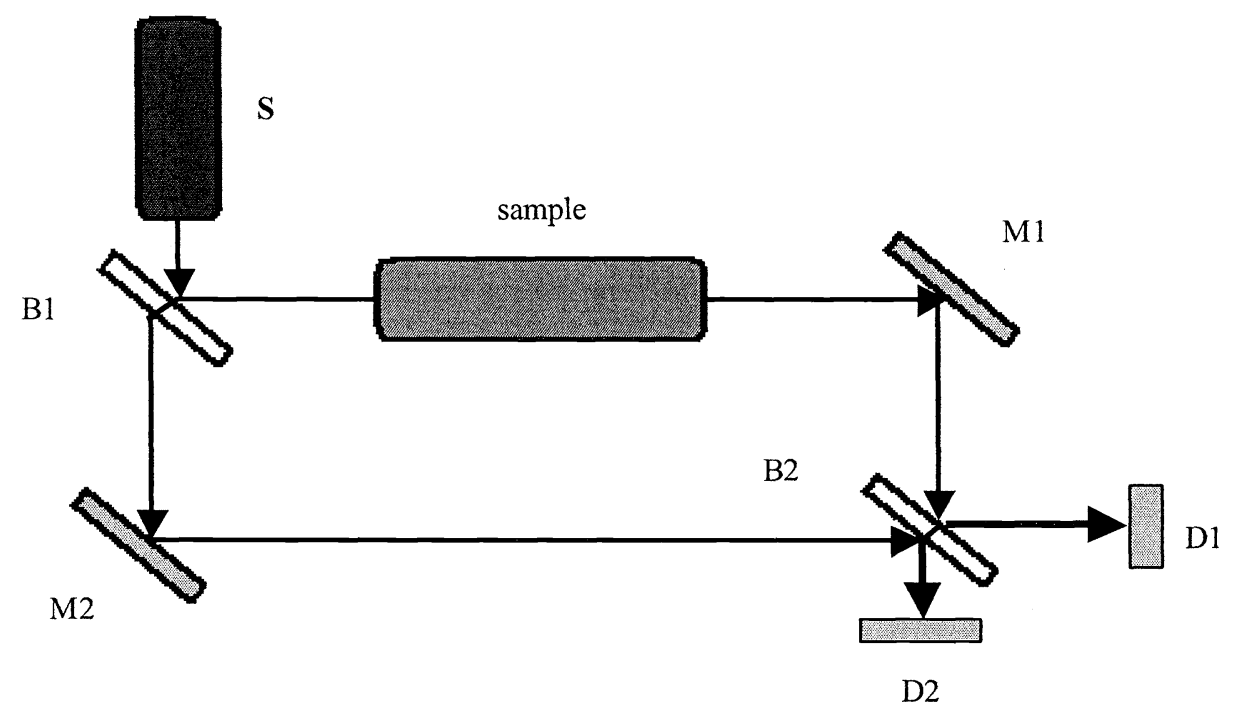

Figure 1. Schematic diagram of the Mach-Zehnder interferometer when used as a refractometer. $\mathrm{S}=$ Source, $\mathbf{B}=$ Beamsplitter, $\mathbf{M}=$ Mirror, $\mathrm{D}=$ Detector

\subsection{Index of Refraction of Water}

The dependence of the index of refraction $\mathrm{n}$ of water on wavelength, temperature and density has recently been studied by Schiebener et $a l^{3}$. Using a large number of experimental data sets published between 1870 and 1990 they arrived at the following formula ${ }^{3}$ :

$$
\frac{n^{2}-1}{n^{2}+2} \frac{1}{\rho_{1}}=a_{0}+a_{1} \rho_{1}+a_{2} T_{1}+a_{3} \lambda_{1}^{2} T_{1}+\frac{a_{4}}{\lambda_{1}^{2}}+\frac{a_{5}}{\lambda_{1}^{2}-\lambda_{\mathrm{uv}}^{2}}+\frac{a_{6}}{\lambda_{1}^{2}-\lambda_{\mathrm{ir}}^{2}}+a_{7} \rho_{1}^{2}
$$

where

$$
\begin{array}{ll}
\rho_{1}=\rho / \rho_{0} & \rho_{0}=1000 \mathrm{~kg} / \mathrm{m}^{3} \\
\lambda_{4}=\lambda / \lambda_{0} & \lambda_{0}=0.589 \mu \mathrm{m} \\
T_{1}=T / T_{0} & T_{0}=273.15 \mathrm{~K}
\end{array}
$$


$\rho$ is the density, $\lambda$ the wavelength, $T$ the absolute temperature, $a_{0}$ to $a_{7}$ are dimensionless coefficients, and $\lambda_{\mathrm{r}}$ and $\lambda_{\mathrm{tv}}$ are the effective infrared and ultraviolet resonances respectively ${ }^{3}$.

The equation holds for the following ranges ${ }^{3}$ :

$\begin{array}{ll}\text { temperature } & 0<T<498 \mathrm{~K} \\ \text { density } & 0<\rho<1060 \mathrm{~kg} / \mathrm{m}^{3} \\ \text { wavelength } & 0.2<\lambda<2.5 \mu \mathrm{m} .\end{array}$

In our calculations we have used the optimized values of the coefficients $a_{0}$ to $a_{7}$ and of $\lambda_{u v}$ and of $\lambda_{\mathrm{ir}}$ that were obtained by Schiebener et $a l^{3}$ :

$$
\begin{array}{ll}
a_{0}=2.43905091 \times 10^{-1} & a_{5}=2.45733798 \times 10^{-3} \\
a_{1}=9.53518094 \times 10^{-3} & a_{6}=8.97478251 \times 10^{-1} \\
a_{2}=-3.64358110 \times 10^{-3} & a_{7}=-1.63066183 \times 10^{-2} \\
a_{3}=2.65666426 \times 10^{-4} & \lambda_{\mathrm{r}}=5.432937 \\
a_{4}=1.59189325 \times 10^{-3} & \lambda_{\mathrm{tv}}=2.292020 \times 10^{-1}
\end{array}
$$

The values for $\rho$ at different $T$ were taken from [4] and were fitted to the polynomial expression:

$$
\rho=b_{0}+b_{1} T+b_{2} T^{2}+b_{3} T^{3}+\ldots
$$

The fit yielded the following values for $b_{0}$ to $b_{3}$ :

$$
\begin{array}{ll}
b_{0}=2.33172322 \times 10^{2} & b_{2}=-1.87742573 \times 10^{-2} \\
b_{1}=6.76473949 & b_{3}=1.56895882 \times 10^{-5}
\end{array}
$$

These are the only coefficients that need to be included in view of our accuracy..

\subsection{Index of Refraction of Air}

The index of refraction $n$ of dry air at $15^{\circ} \mathrm{C}$ and a pressure of $1.013 \times 10^{5} \mathrm{~Pa}$ has been calculated from the expression $^{4}$

$$
(n-1) \times 10^{8}=8342.13+240603\left(130-\sigma^{2}\right)^{-1}+15997\left(38.9-\sigma^{2}\right)^{-1}
$$

where $\sigma=1 / \lambda_{\mathrm{ac}}$ and $\lambda_{\mathrm{rac}}$ is the wavelength in vacuum of the laser beam in $\mu \mathrm{m}$. This equation is valid for wavelengths between $200 \mathrm{~nm}$ and $2 \mu \mathrm{m}$. For pressures and temperatures different from the indicated values, the value of $(n-1)$ has to be multiplied by ${ }^{4}$

$$
\frac{p\left[1+p(61.3-T) \times 10^{-10}\right]}{96095.4(1+0.003661 T)}
$$

where $p$ is the pressure (in $\mathrm{Pa}$ ) and $T$ is the temperature (in ${ }^{\circ} \mathrm{C}$ ). We have used the equations (5) and (6) to calculate the index of refraction for air.

\section{Experimental Set-up}

The physical set-up of the fiber-optic version of the Mach-Zehnder interferometer is shown in figure 2. A $10 \mathrm{~mW}$ HeNe laser (Uniphase $1135 \mathrm{P}$ ) with a linearly polarized output at $632.8 \mathrm{~nm}$ is used as light source. The laser output is split into two beams using a bi-directional coupler (Thor Labs, custom made) employing single mode fibers. The laser signal is coupled to the input fiber of the coupler using a single mode fiber launcher (Thor Labs KT210/M). The output fiber ends are placed in fiber mounts and serve as sources of the interfering beams, which are recombined in a $50 / 50$ plate beamsplitter ( 2 inch in diameter). All 


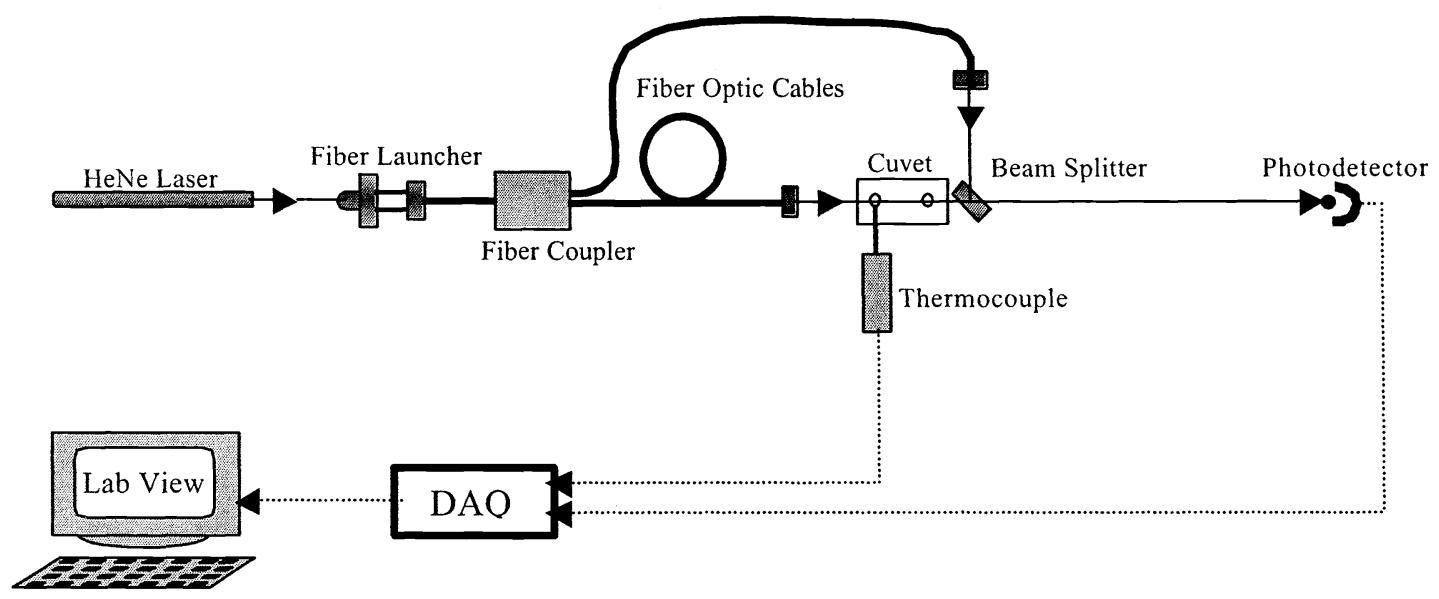

Figure 2. The fiber optic version of the Mach-Zehnder interferometer used as a refractometer.

elements from the laser up to the beamsplitter are mounted on a matrix plate to ensure optimum stability of the system.

For the relative measurements of the index of refraction of water, a $50.0-\mathrm{mm}$ long optical glass cuvet is filled with water and placed in the open air space between the beamsplitter and one of the fiber arm ends. A nichrome wire is wound uniformly around the cuvet in order to allow controlled and uniform heating. The temperature measurements have been automated. A calibrated (accuracy $\pm 0.1{ }^{\circ} \mathrm{C}$ ) thermocouple was used as a temperature sensor. The output of this sensor is sent to a computer via a Data Acquisition card (DAQ).

The detection of the fringe shifts and hence the change in optical path length was also automated. A photodetector placed at the center of the interference pattern detects the variations in intensity, which are then stored in a computer using a DAQ card. A program coded in LabVIEW counts the number of fringes over which the pattern shifts.

For the absolute measurements of the index of refraction of air, the cuvet is evacuated to a pressure of less than 5 mbar using a rotary pump. After evacuating the cuvet, the air is slowly let back in using a needle valve, during which process the fringe shift is recorded.

\section{Results and Discussion}

\subsection{Interference Pattern}

A stable interference pattern with good contrast was quite easily obtained. The overlap of the two beams at the beamsplitter turned out to be the most critical factor. The distances between the fiber ends and the beamsplitter were not critical in obtaining a good quality pattern. A rigid fixation of all optical elements to the matrix plate is essential for a good stability of the interference pattern. The fixation to the matrix plate enables a setting of the fiber mounts in such way that the two beams are mutually perpendicular. The insertion of the cuvet did not affect the quality and stability of the pattern. The visibility $\mathrm{V}$ of the interference pattern was calculated from':

$$
\begin{aligned}
& \mathrm{V}=\underline{I}_{\max }+\mathrm{I}_{\min } \\
& \mathrm{I}_{\max }-\mathrm{I}_{\min }
\end{aligned}
$$

where $I_{\max }$ and $I_{\min }$ represent the maximum and the minimum intensities. Using the values for $I_{\max }$ and $I_{\min }$ as measured by the photodetector, we obtained a value of $63 \%$ for $\mathrm{V}$, enough to allow proper counting of the fringe shift. This result was quite easily obtained without a long process of optimizing various parameters. Figure 3a shows the so-called "bull's eye" type of interference pattern. This pattern was obtained from the interferometer and captured using a charged coupled device (CCD) camera. 


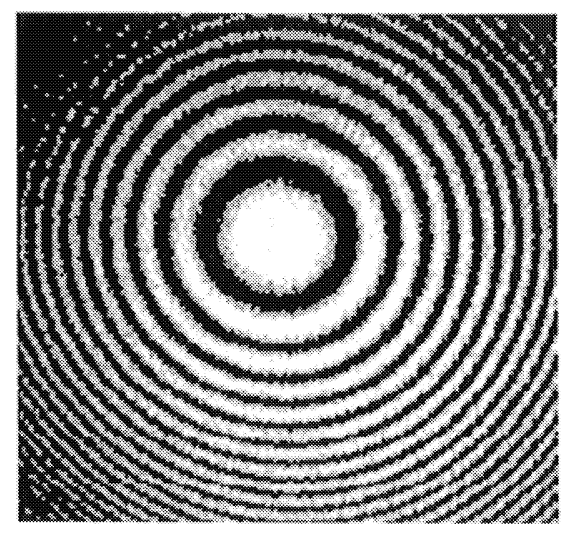

(a)

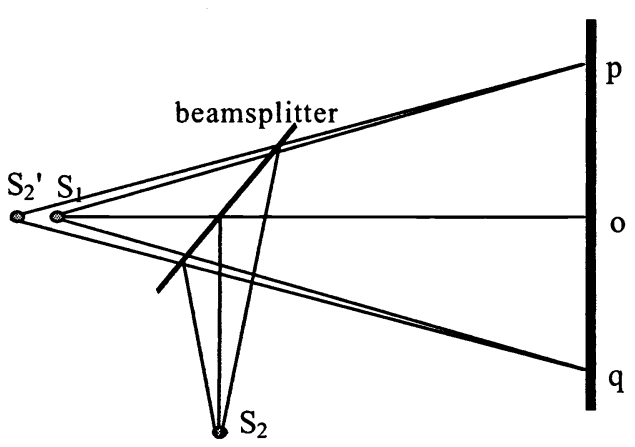

(b)

Figure 3: a)The 'bulls-eye' type of interference pattern. b) optical ray tracing of the interferometric set up

The geometry behind the formation of the circular interference pattern from the Mach-Zehnder interferometer is illustrated in figure $3 \mathrm{~b}$. In the fiber-optic version of the interferometer, the points $\mathrm{S}_{1}$ and $\mathrm{S}_{2}$ represent the ends of the fiber arms which serve as the coherent sources of the two interfering beams. The point $S_{2}$ forms a virtual image $S_{2}$ ' relative to the beam splitter. A pattern of circular dark and bright fringes is obtained is obtained after the two beams from $S_{1}$ and $S_{2}$ ' start to interfere at the beamsplitter.

\subsection{Polarization}

The polarization of the interfering beams is an important factor when considering the visibility of the interference pattern. The laser beam is $99 \%$ linearly polarized. When using single mode fibers for the transmission of a beam, it is expected that the polarization is preserved when using relatively short lengths of fiber. We have checked the polarization of the two beams emerging from the fiber ends by passing the beams through a polarizer. The results are shown in figure 4, where the power behind the polarizer is shown as function of the polarizing angle $\theta$. The results indicate that the two beams coming from the fiber ends are nearly completely linearly polarized. So in our case the single mode fibers act as polarization preserving fibers. It is also seen that the two beams are slightly different in power, although the bidirectional coupler is supposed to make a 50-50 split of the incoming beam. Arranging for equal powers in the two beams may further improve the visibility. But a visibility of $63 \%$ was sufficient in the application as a refractometer.

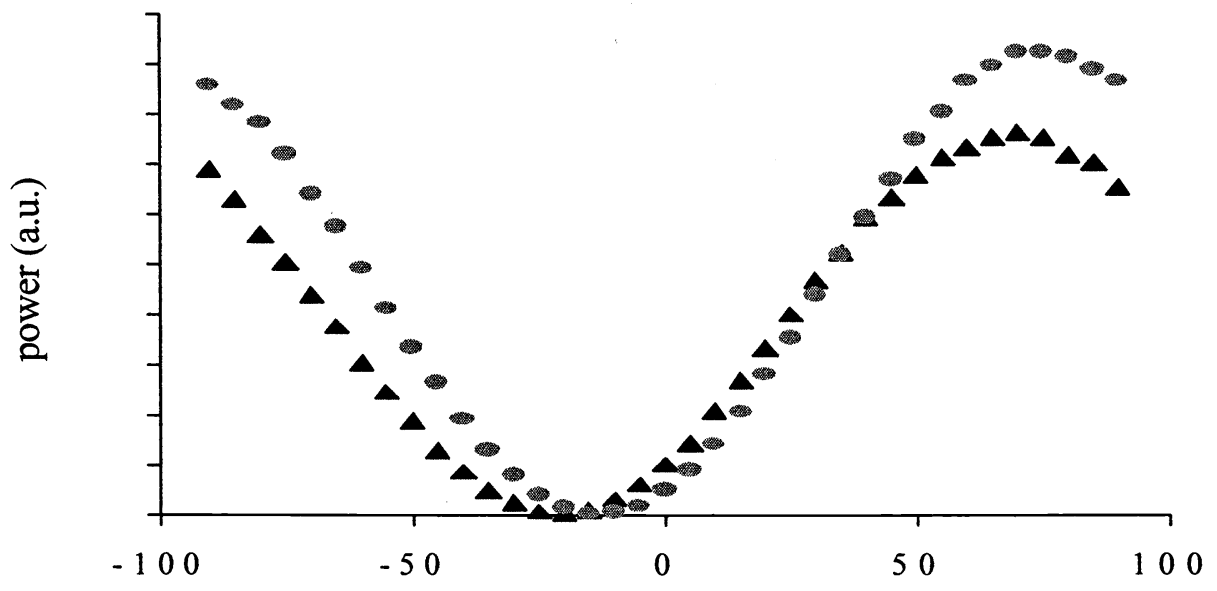

Figure 4 Polarization of the beams from the optical fibers 


\subsection{Relative Measurements of the Index of Refraction of Water}

We have measured the change in the index of refraction of water as a function of temperature. Heating of the water from $25{ }^{\circ} \mathrm{C}$ to $75^{\circ} \mathrm{C}$ resulted in a total shift of 645 fringes. Going through the same interval in opposite direction (cooling) resulted in a total shift of 650 fringes. This difference is attributed to nonuniformities in the heating and cooling. This five-fringe difference was taken as the error in the total fringe shift when going from $25^{\circ} \mathrm{C}$ to $75^{\circ} \mathrm{C}$. We have investigated several possible systematic errors. The effect of the linear expansion of the glass cylinder when heated can be neglected. The calculated maximum change in the length of the glass cylinder due to the heating is $2 \times 10^{-5} \mathrm{~m}$ (using a coefficient $\alpha=0.9 \times 10^{-5}$ for the linear expansion ${ }^{5}$ ). Another possible error, a change in the index of refraction of the glass due to a changing temperature is also small. Heating the cuvet filled with air from $25^{\circ} \mathrm{C}$ to $75^{\circ} \mathrm{C}$, resulted in a shift of only two fringes. Our error analysis shows that both systematic effects can be neglected. The error in the results can be attributed to errors in the measurement of the fringe shift. The percentage error in the results for $n-n_{\mathrm{o}}\left(\right.$ at $\left.75^{\circ} \mathrm{C}\right)$ is estimated at $1 \%$.

Shown in figure 5 are the curve computed using equations (2), (3) and (4), and the experimental results. We observed a maximum difference of $1 \%$, which is in good agreement with the accuracy of our measurements.

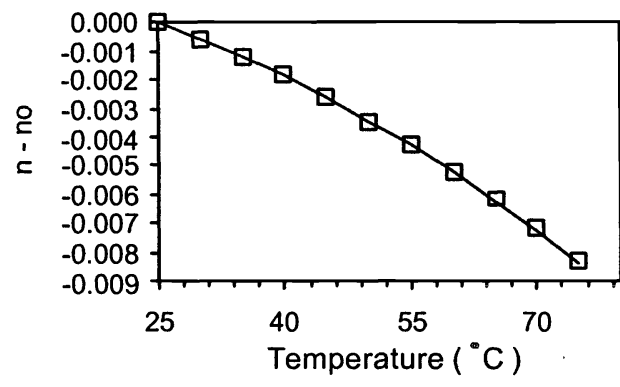

Figure 5. The experimental values of the index of refraction of water ( $(\square)$ as a function of temperature compared with the values calculated from references [3] and [4] (solid line).

\subsection{Absolute Measurements of the Index of Refraction of Air}

We have done absolute measurements on the index of refraction of air at $p=1.013 \times 10^{5} \mathrm{~Pa}$ and $T=21.0$ ${ }^{\circ} \mathrm{C}$. The fringe shift as appearing on the computer screen is shown in figure 6 . The maxima and minima represent bright and dark fringes respectively. Going from one maximum to next maximum via a minimum corresponds to a shift of a single fringe. The visibility of $63 \%$ is enough to enable proper counting.

We have counted a shift of 21.0 fringes going from vacuum $\leqslant 5 \mathrm{mbar})$ to atmospheric pressure. The result represents the average from a series of measurements. From the series of results, we estimate the error at \pm 0.5 fringes. Using equation (1), we obtained a value for the index of refraction of air at $21.0^{\circ} \mathrm{C}$ and at pressure of $1.013 \times 10^{5} \mathrm{~Pa}$, equal to $1.000267 \pm 0.000007$. This result is in good agreement with the calculated value of 1.000271 . The accuracy of our results up to six digits compares well with the accuracy of results given in literature ${ }^{4}$. 


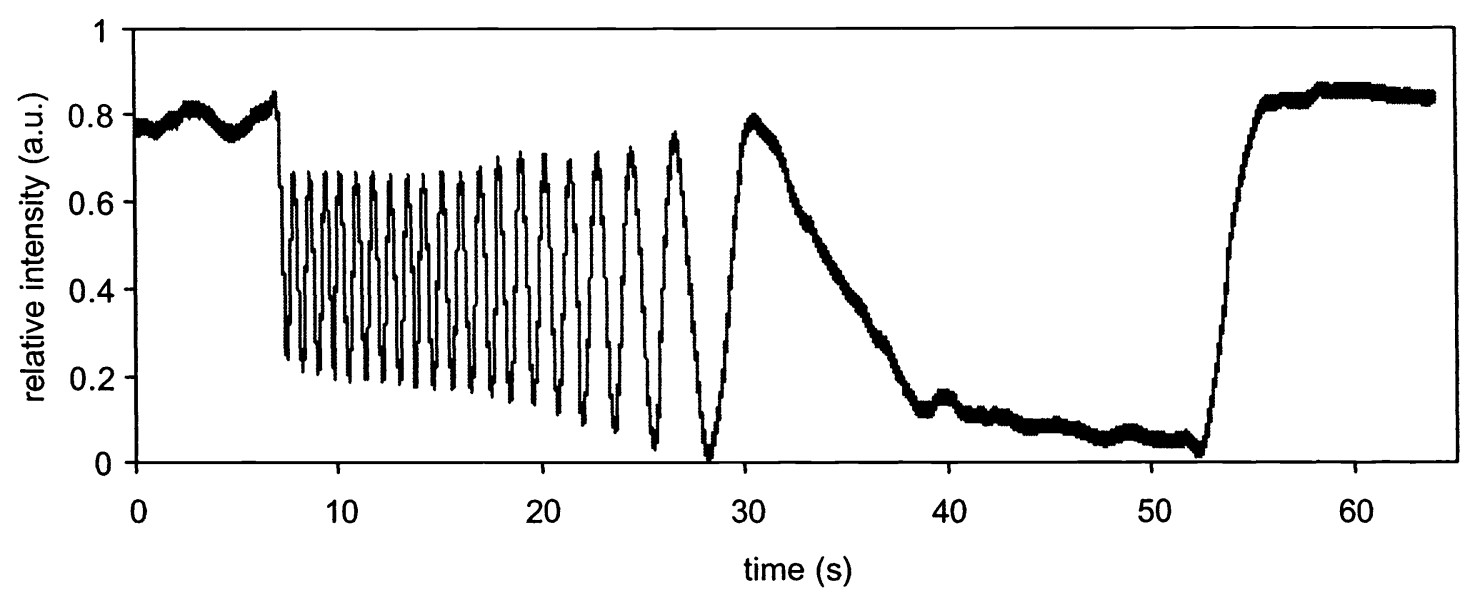

Figure 6. Signal from the photodetector as observed when air is let into the evacuated cell. The maxima and minima represent the variation from bright to dark fringe respectively. Going from one minimum to the next minimum via a maximum or one maximum to the next maximum via a minimum corresponds to a single fringe shift.

\section{Conclusion and Discussion}

A stable interference pattern with a very good visibility could quite easily be obtained using the fiber optic version of the Mach-Zehnder interferometer. This stable and sensitive version of the interferometer makes it a very good device in several applications. One of them is the use as a refractometer as described above. The use of fibers reduces the sensitivity for external disturbances and makes it easier to handle the interferometer. The recombination of the two beams still occurs in the open and allows for the insertion of samples in the arms of the instrument.

We have done relative measurements on the index of refraction of water with high accuracy. An accuracy of up to six decimal digits was attained for the absolute measurements in air. The automation of the measurement of the fringe shift increases the reliability of the counting process and makes it quite easy to do measurements involving shifts over hundreds of fringes.

The experience over the past two years at the University of San Carlos has shown that the BS students can work with the instrument described above. Initially it was used for special projects but by now we are using it for regular laboratory classes, where it can be applied in a variety of experiments in optics and optoelectronics. The relatively low cost of the instrument (our total expenditure was about US\$ 2,500.-) brings it within the reach of many laboratories.

\section{Ackowledgements}

The authors greatly acknowledge the Netherlands University Foundation for International Cooperation (NUFFIC) for the financial support to this project.

\section{References}

[1] F.A. Jenkins and H.E. White, Fundamentals of Optics, Mc-Graw-Hill, 1976.

[2] M.A. Flores et al, A Fiber Optic Version of the Mach-Zehnder Interferometer for Use in the Undergraduate Physics Laboratory, Proceedings of the $18^{\text {th }}$ SPP, Physics Congress, October 2000, p. 271-273.

[3] P. Schiebener et al, Refractive Index of Water and Steam as a Function of Wavelength, Temperature and Density, J. Phys. Chem. Ref. Data, Vol. 19,No.3, 1990, p. 677-717.

[4] D. R. Lide, CRC Handbook of Chemistry and Physics, CRC Press Inc., 1992 
[5] H. D. Young and R. A. Freedman, University Physics, Addison Wesley, 1999, p. 479.

[6] A.S. Flores et al., Measurements of the Index of Refraction of Air and Water Using a Mach-Zehnder interferometer, Proceedings of the $19^{\text {th }}$ SPP, Physics Congress, October 2001, p. 287-290. 